\title{
Targeting transient phenomena with e-VLBI
}

\section{Zsolt Paragi*}

JIVE, MTA Research Group for Physical Geodesy and Geodynamics

E-mail: zparagi@jive.nl

\section{Huib Jan van Langevelde}

JIVE, Sterrewacht Leiden, Leiden University

E-mail: langevelde@jive.nl

\section{Arpad Szomoru}

JIVE

E-mail: szomoruejive.nl

e-VLBI is a technique under development that allows real-time correlation of VLBI data! At the EVN there have been routine e-VLBI tests and science observations in the last 18 months. We will briefly introduce the status of the e-EVN, and show early e-VLBI results. Potential use of e-VLBI for various classes of transient or rapid variable sources will be described.

Bursts, Pulses and Flickering:Wide-field monitoring of the dynamic radio sky June 12-15 2007 Kerastari, Tripolis, Greece

${ }^{*}$ Speaker.

$\dagger$ e-VLBI developments in Europe are supported by the EC DG-INFSO funded Communication Network Developments project 'EXPReS', Contract No. 02662 (http://www.expres-eu.org/). The European VLBI Network (http://www.evlbi.org/) is a joint facility of European, Chinese, South African and other radio astronomy institutes funded by their national research councils. 


\section{What is e-VLBI}

In electronic very long baseline interferometry (e-VLBI) the data from distant radio telescopes are streamed to the central processor through optical fibres, and correlated in real-time. One great advantage of this technique currently under development, is that the observations are no longer dependent on the availability of disk space at the telescopes, allowing long observations at high data rates. It is envisioned that eventually data rates up to several Gbit/s/telescope will be achievable in the future, increasing the sensitivity of present radio arrays considerably. Another new aspect of e-VLBI is that the results from the observations are immediately available, from which certain science projects may benefit. These are most likely programmes targeting transient or rapidly variable sources. Once the e-VLBI capability is coupled with quick array reaction time and flexible scheduling for follow-up observations, a new era of VLBI transient science will begin. With the advent of new survey telescopes (see these proceedings for several examples) the number of discovered transients, likely including new source classes is expected to increase dramatically.

\section{2. e-VLBI at the EVN}

Here we will describe briefly the present e-VLBI network at the EVN (see Fig. 1). There have been e-VLBI developments in Australia (see Tzioumis, these proceedings), Japan and USA. The European programme is based on the Mark5A disk recording system, developed in Haystack (http://www.haystack.edu/tech/vlbi/mark5/). The e-EVN in mid-2007 consisted of seven telescopes: Cambridge (or Darnhall), Jodrell Bank (MkII), Medicina, Metsahovi, Onsala, Torun, and Westerbork (phased array). Up-to-date information on the available stations and the frequecny bands offered for e-VLBI observations can be found on the EVN e-VLBI web pages (http://www.evlbi. org/evlbi/e-vlbi_status.html). All of these can be connected to the EVN MarkIV Correlator at JIVE through $1 \mathrm{Gbit} / \mathrm{s}$ links. In the early phase of test and science operations (early 2006) we achieved $128 \mathrm{Mbit} / \mathrm{s}$ data rates using the TCP protocol (http://www.faqs.org/rfcs/rfc793.html). After hardware upgrades and data transfer tuning this improved to $256 \mathrm{Mbit} / \mathrm{s}$, and occasionally $512 \mathrm{Mbit} / \mathrm{s}$. The latest tests show that $512 \mathrm{Mbit} / \mathrm{s}$ or higher data rates are sustainable for long observations with UDP-based transfer protocols (http://www.faqs.org/rfcs/rfc768.html). Several new telescopes are going to join the array soon: the $100 \mathrm{~m}$ Effelsberg, the $40 \mathrm{~m}$ Yebes, the $25 \mathrm{~m}$ Shanghai, and the $300 \mathrm{~m}$ Arecibo telescopes, partially funded by the EXPReS project. Running at $512 \mathrm{Mbit} / \mathrm{s}$ this will result in a very competitive array in sensitivity and resolution.

Initially science observations were carried out at fixed dates when e-VLBI tests were done. Proposals were accepted up two two weeks before the tests. Once the operations became more robust, it was realized that the proposal rules must be changed in order to better exploit the eEVN capabilities. As from 1 June 2007, e-VLBI proposals must be submitted by the normal EVN deadlines. However a new "triggered proposal" class has been defined. In this type of proposal one may request observations of a class of objects, if a certain triggering criterion is met up to $24 \mathrm{~h}$ before the observations. It is expected that in the coming years e-VLBI will no longer be restricted to the fixed test dates, and closely spaced monitoring observations will be possible - given that the science case is strong enough to overrule schedules of individual telescopes. 


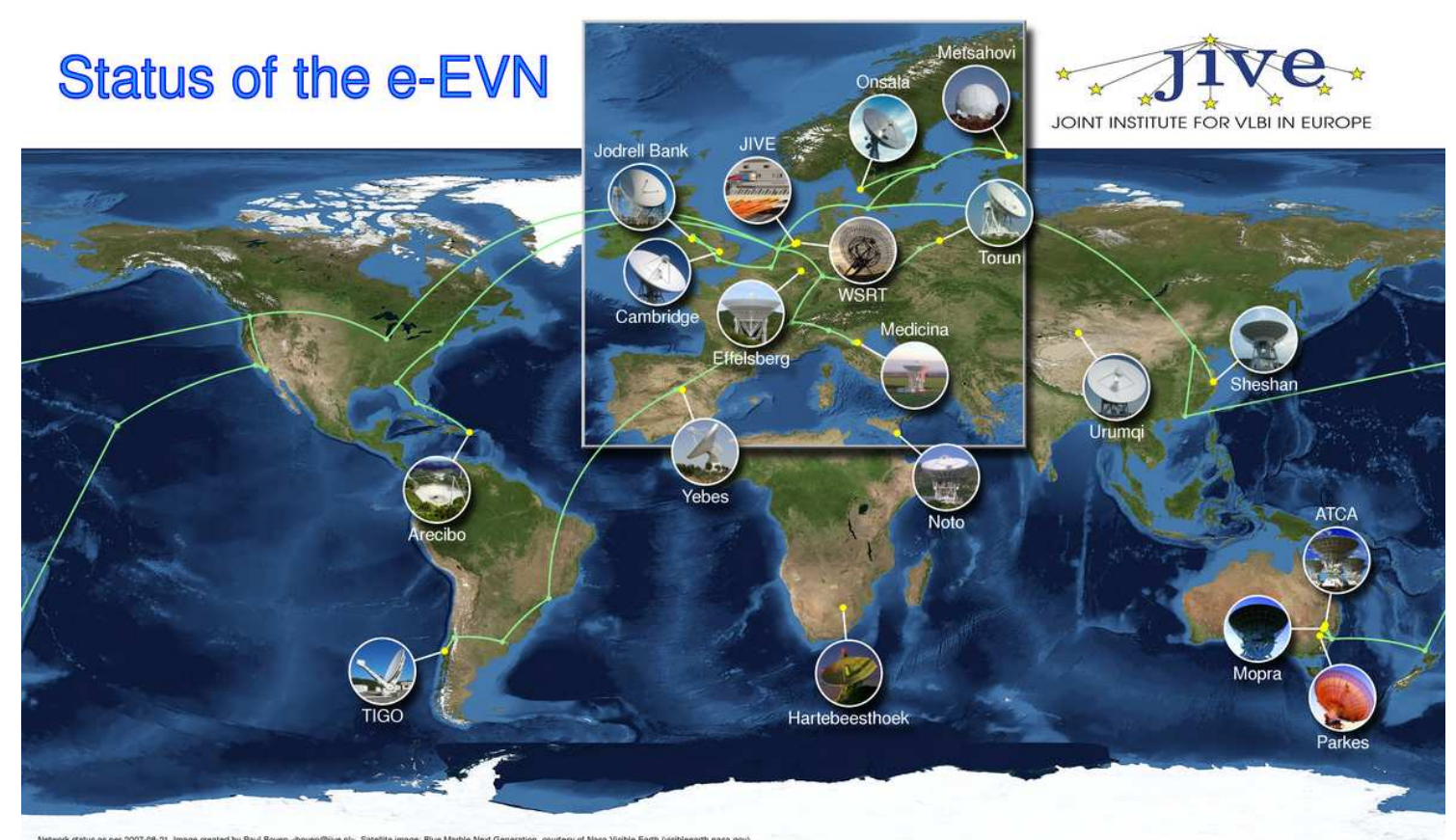

Figure 1: Telescopes participating in the EXPReS project

\section{Galactic transients}

Galactic transients evolve on timescales significantly shorter $(\sim$ days $)$ than extragalactic transients. Because of the short timescales involved, one may argue that real-time correlation offered by e-VLBI is less crucial; it is the array response time and flexibility that will determine the success of the target of opportunity (ToO) observations. While this is mostly true, e-VLBI has a number of advantages. Telescope participation in a ToO projects may depend on availability of recording media, while e-VLBI is not limited by this. Another advantage is the real-time monitoring of telescope performance, which guarantees the success of observations. This is important for the EVN where VLBI observations are limited to three observing sessions in a year, and immediate change from a single dish program to VLBI involves certain risks. Especially, in the future e-VLBI will be very important for observations with ad-hoc arrays on global scales. Moreover, with e-VLBI the data are immediately available for the astronomer for further processing. Quick analysis tools (including a full data analysis pipeline) have been developed at JIVE recently. Using the rapid feedback from the array, one may plan follow-up observations more efficiently, or even trigger further observations with other telescopes based on the VLBI results themselves (Rushton and Spencer, these proceedings).

Microquasars -and in a broad sense, all X-ray binary systems- are some of the best candidates for e-VLBI observations. These systems regularly show outbursts, but only a handful of these events have been followed-up by VLBI. Fundamental questions are the bulk jet Lorentz-factor in these systems (often difficult to determine also because the source distances are not known 
accurately), similarity or difference of jet properties between black hole and neutron star XRBs, and whether the Lorentz-factor changes during accretion state changes. To answer these questions many more observations are necessary. How to trigger e-VLBI observations on such very short notice is a difficult problem. By the time the flare brightens sufficiently, the source has already expanded significantly. The early phases of jet evolution will thus be hidden. A promising approach is to find an optical/IR trigger that precedes the flare several days (see Russel, these proceedings). This would give the opportunity to observe the burst from the radio quenching phase, which has never been observed with VLBI.

Microquasar jet polarization properties have not been fully explored so far with VLBI. Note that up to date, there was a single experiment in which mas-scale polarization has been detected (to our knowledge); that was an e-EVN observation of Cyg X-3 (Fig. 2). The e-EVN array together with the phased array Westerbork telescope will be a powerful tool to analyse jet polarization properties. Using the WSRT synthesis array data (recorded parallel to the VLBI run) one may calibrate in the VLBI data the level and PA of the polarization in the calibrators and in the target. Moreover, because the WSRT has linear feeds, measuring circular polarization will not face the extreme difficulties we normally have in VLBI observations, namely that we do not know a-priori the extent of $\mathrm{CP}$ in any of our calibrators.

So far only a tiny fraction of the XRB population has been detected in the radio. Most of these sources show activity on the mJy level, ruling out the possibility of triggering based on bright flares. A larger fraction of this population will be available for further study from early next year, when the 100m Effelsberg telescope and Arecibo join the e-EVN. Imaging of sources at the 100-1000 $\mu \mathrm{Jy}$ level will be possible routinely. With e-VLBI, it will be possible to observe a sample of these sources initially, and follow-up immediately the ones that show interesting morphology at the first epoch. This could be an alternative method to trigger further VLBI monitoring observations of these sources.

\section{Extragalactic transients}

Although extragalactic transients evolve more slowly, e-VLBI could be still important for ToO observations with inhomogeneus arrays, as mentioned above. An interesting class of highly variable objects (not transient in the classical sense) are ultra-luminous X-ray sources (ULXs). There has been a debate whether these systems are powered by intermediate-mass black holes (IMBH, [2]), or they are super-Eddington accreting microquasars [e.g. 3]. While the majority of these sources are likely to be related to stellar-mass objects [4], the accretion disk properties suggest that some of them may be IMBHs [5]. Future radio observations of these systems could help to resolve this debate [6]. If caught in an a radio-active state in ToO e-VLBI observations, one could pinpoint the location of the ULX which would help to identify the optical counterpart.

In fact there is one group of sources that may evolve on timescales requiring e-VLBI observations: GRB jets and the likely related type Ib/c supernovae. It has been suggested that the ultra-relativistic jets produced by these systems should be resolvable in high-frequency VLBI observations $[7,8]$. This would be extremely important because there has been little direct imaging support so far of the proposed model of GRBs [but cf. 9,10,11]. Whether one should monitor these systems on a weekly basis or every 6 months depends on the source expansion rate, which 

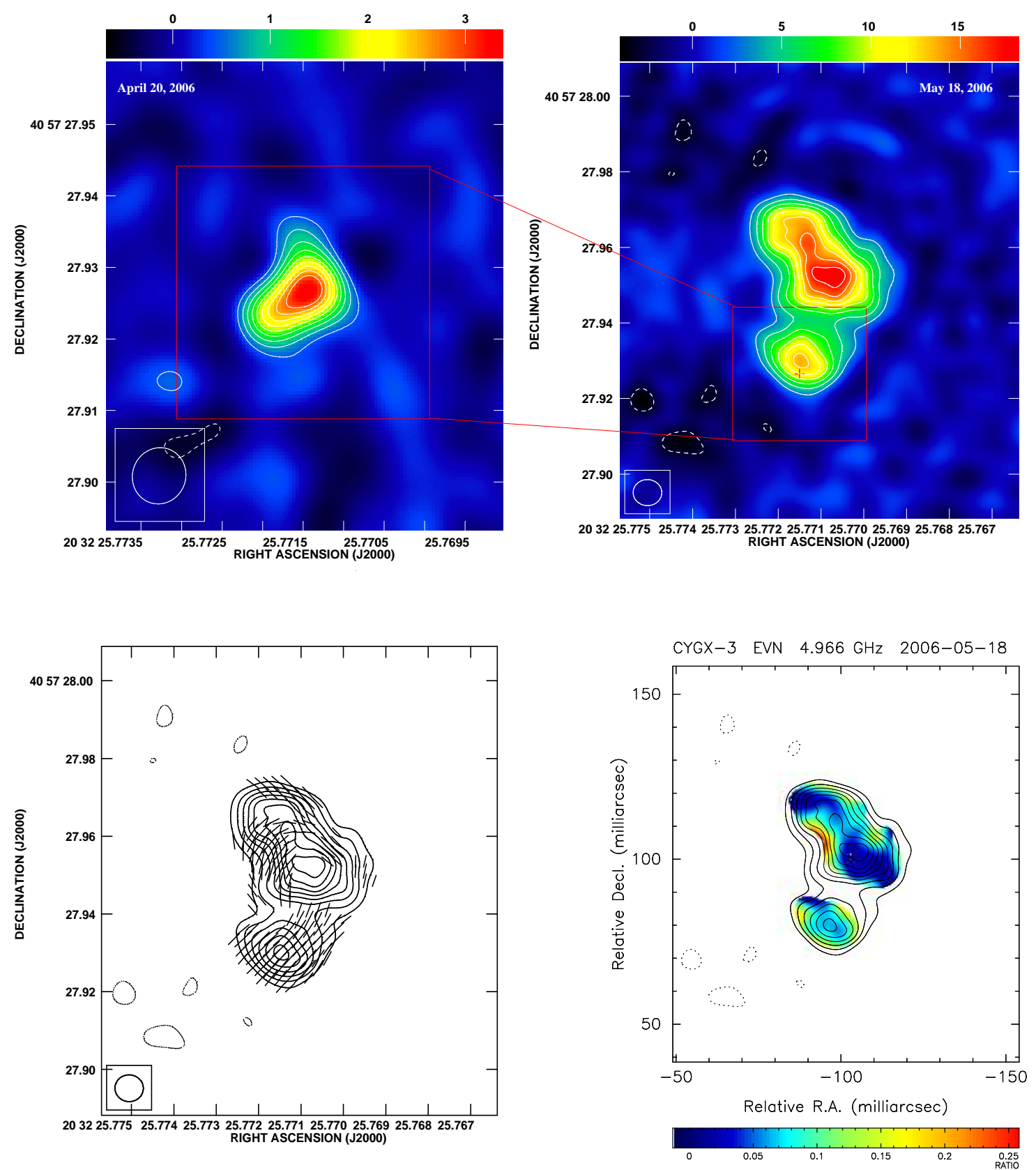

Figure 2: e-EVN images of Cyg X-3 in the stages after outbursts in 2006. The upper panel shows the total intensity image. The lower left panel shows the (PA-uncorrected) linear-polarization map, the lower-righ panel the percentage of linear-polarization in the source. Figure courtesy Valeriu Tudose 

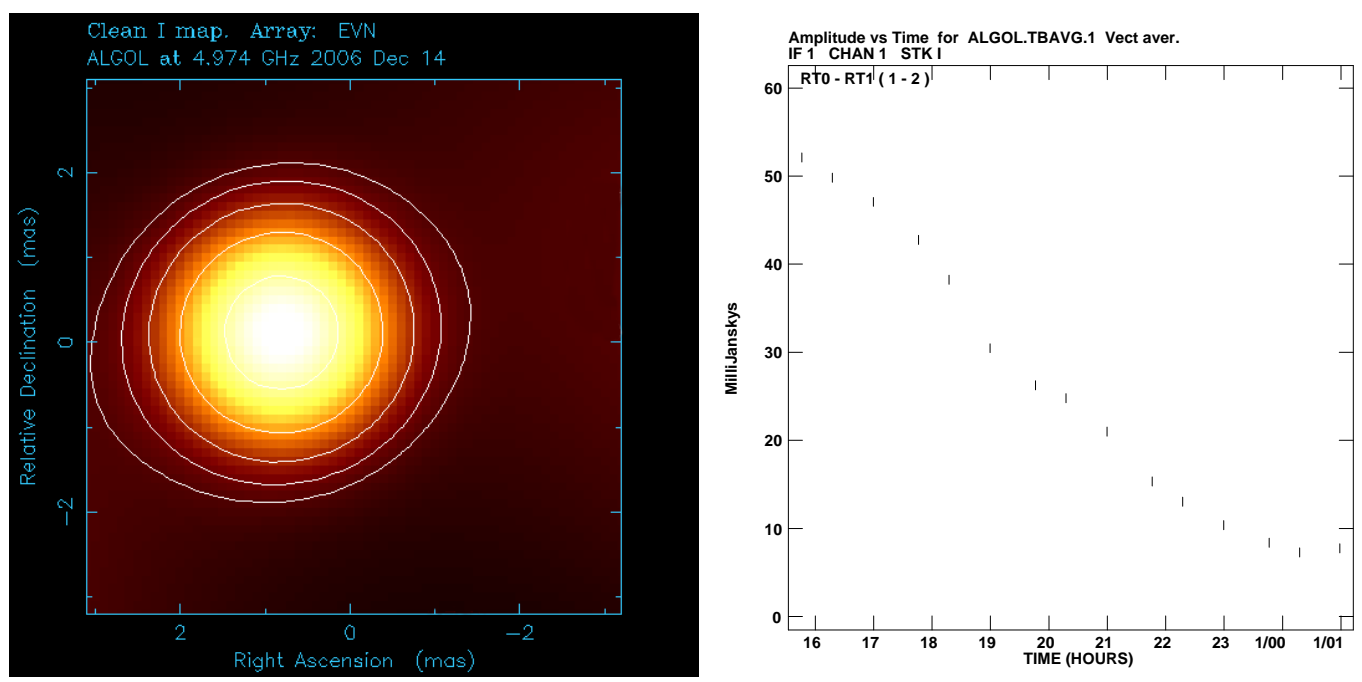

Figure 3: e-EVN total intensity image (left) and simultaneous WSRT lightcurve (right) of Algol on 14 December 2006. The system remained unresolved in this run, however the spectral properties suggested that the flare location was not coincident with the normal gyrosynchrotron lobes (the CP peak was about 2 mas to the West of the I peak). These events have so short timescales that dedicated observations are not possible without e-VLBI and dynamic scheduling during the observations (Csizmadia et al., in prep.)

could easily exceed 1 milliarcsecond/month for Local Group SNe, if there is an underlying relativistic jet oriented in our line of sight (Ramirez-Ruiz, priv. comm.). High resolution and sensitive e-VLBI observations would be able to address this problem.

\section{Variable stars}

Variable radio stars (see Oosten, these proceedings) have rarely been observed with VLBI before because of their relative faintness. However present VLBI arrays can easily observe sources at the 100-1000 $\mu \mathrm{Jy}$ levels, which opens up the possibility to observe variable stellar sources in much larger numbers. One challenge is the variability timescale, which could be hours or shorter. The role of e-VLBI may be here to be able to select sources from a sample, that show peculiar activity during the observations. Once adaptive scheduling is available (work is going on this in JIVE), one could efficiently select the targets that are interesting at that very moment, and continue observations only on that target.

To demonstrate this, we show an e-EVN image and the Westerbork Synthesis Radio Telescope lightcurve of Algol (Fig. 3), observed in December 2006 by Csizmadia et al. (in prep.). Algol underwent a flare during the e-VLBI run, that faded on a timescales of hours. In the same time the circular-polarization properties significantly changed in the system. The origin of these flares in Algol systems is not known at present. Future dedicated observations of these events may only be possible with a high-resolution e-VLBI array, that is capable of changing source schedule during the observations. 


\section{Conclusions}

There are promising developments in the field of e-VLBI. The main advantage will eventually be a significantly increased observing bandwidth, but certain science applications may benefit from the quick science turnaround time as well.

\section{References}

[1] V. Tudose, R.P. Fender, M.A. Garrett, J.C.A. Miller-Jones, Z. Paragi, R.E. Spencer, G.G. Pooley, M. van der Klis, M., A. Szomoru, Mon. Not. R. Astron. Soc. 375, L11 (2007).

[2] E.J.M. Colbert, R. F. Mushotzky, Astrophys. J. 519, 89 (1999).

[3] S. Fabrika, A. Mescheryakov, Proc. IAU Symposium 205, 268 (2001).

[4] A. R. King, M. B. Davies, M. J. Ward, G. Fabbiano, M. Elvis, Astrophys. J. 552, L109 (2001).

[5] J. M. Miller, A. C. Fabian, M. C. Miller, Astrophys. J. 614, L117 (2004).

[6] Z. Paragi, M.A. Garrett, A.D. Biggs, A.D., Proceedings of Science, PoS(8thEVN)016 (2007)

[7] B. Paczyński, Acta Astronomica 51, 1 (2001)

[8] J. Granot, A. Loeb, Astrophys. J. 593, L81 (2003).

[9] G. Taylor, D.A. Frail, E. Berger, S.R. Kulkarni, Astrophys. J. 609, L1 (2004).

[10] G. Taylor, E. Momjian, Y. Pihlström, T. Ghosh, C. Salter, Astrophys. J. 622, 986 (2005).

[11] Y. Pihlström, G. Taylor, J. Granot, S. Doeleman, Astrophys. J. 664, 411 (2007). 